\title{
Elementos de la calidad del servicio en las empresas mixtas venezolanas
}

\author{
Elements of the quality of the service in the venezuelan mixed companies \\ Elementos da qualidade do serviço nas sociedades mistas venezuelanas
}

Recibido: enero 2019

Arbitrado: febrero 2019

Publicado: mayo 2019

\author{
《 Alexandra Rangel \\ ingalexandrarangel@hotmail.com \\ ORCID: 0000-0002-5730-5441 \\ Universidad del Zulia, Venezuela
}

RESUMEN

Bajo una metodología descriptiva, con diseño no experimental, transeccional y de campo, en la presente investigación, se determinaron los elementos de la calidad del servicio en las empresas mixtas venezolanas. La población quedó conformada por los gerentes, líderes y supervisores adscritos a las gerencias de contratación de las empresas mixtas de la industria petrolera del municipio Maracaibo, a quienes se les aplico una encuesta mediante un cuestionario, conformado por 12 ítems con escala Likert. La validez se realizó a través del juicio de expertos, y para calcular su confiabilidad se empleó el método Alfa de Cronbach, resultando 0,91. El análisis de los datos se realizó mediante la estadística descriptiva, con base en la media aritmética, apoyada en las frecuencias relativas. Los resultados demostraron alta presencia del costo de la mala calidad, posición del mercado, cultura de la calidad y operación del sistema, como elementos de la calidad del servicio.

Palabras clave: Calidad del servicio; costo de la mala calidad; cultura de la calidad; operación del sistema, posición del mercado
Using a descriptive methodology, with a non-experimental, cross-sectional and field design, in this research, the elements of service quality in Venezuelan mixed companies were determined. The population was made up of the managers, leaders and supervisors assigned to the hiring managers of the mixed companies of the oil industry of the Maracaibo municipality, to whom a survey was applied by means of a questionnaire, made up of 12 items with a Likert scale. Validity was carried out through expert judgment, and the Cronbach's Alpha method was used to calculate its reliability, resulting in 0.91 . Data analysis was performed using descriptive statistics, based on the arithmetic mean, supported by relative frequencies. The results showed a high presence of the cost of poor quality, market position, quality culture and system operation, as elements of service quality.

Key words: Quality of service; poor quality cost; quality culture; system operation, market position

\section{RESUMO}

Sob uma metodologia descritiva, com desenho não experimental, transversal e de campo, na presente investigação, foram determinados os elementos de qualidade de serviço nas joint ventures venezuelanas. A população foi constituída pelos gestores, dirigentes e supenvisores lotados nas áreas de contratação das empresas mistas da indústria do petróleo do município de Maracaibo, aos quais foi aplicada uma pesquisa por meio de um questionário, composto por 12 itens em escala Likert. A validade foi realizada por meio do julgamento de especialistas, sendo utilizado o método Alfa de Cronbach para calcular sua confiabilidade, resultando em 0,91. A análise dos dados foi realizada por meio de estatística descritiva, com base na média aritmética, suportada por frequências relativas. Os resultados evidenciaram alta presença do custo da má qualidade, posicionamento no mercado, cultura de qualidade e operação do sistema, como elementos da qualidade do serviço.

Palavras chave: Qualidade de serviço; custo de baixa qualidade; cultura de qualidade; operação do sistema, posição de mercado 


\section{INTRODUCCIÓN}

$\mathrm{H}$ oy en día, las empresas se han dedicado en abarcar una serie de aspectos que determinan la calidad de servicio y que no solo se enmarca en la atención, sino también en lo referente a instalaciones, tiempos de respuestas, cercanía, centros de atención, variedad de productos, entre otros, así como lo afirma Grande (2005), los consumidores de servicios buscan precios bajos, servicios personalizados, de excelente calidad, exigencias cada vez mayores y más específicas en el futuro.

Esta manera de atender o complacer a la que se han dedicado las organizaciones proveedoras de servicio va más allá de satisfacer necesidades externas, el desarrollo de todos los aspectos anteriormente señalados se enfocan a la par en satisfacer necesidades internas $y$, esto se traduce en crear elementos diferenciadores ante el ramo en que se está involucrado, que no es otra cosa que la competencia a la que se debe enfrentar cuando existe en el mercado una amplia presencia de empresas que proveen el mismo servicio.

Sobre ello, Ivancevich y otros (2005), señalan que la competencia es ahora tan intensa que estimula la adopción de un enfoque más agresivo en la gestión de personal y en la mejora de la calidad, si se quiere ser competitivo se han de asignar la calidad, tiempo y atención debidos y, ha de mantenerse en la organización un ambiente propicio para la introducción de innovaciones.

En este contexto, se visualiza que la calidad de servicio es un término en el que actualmente las empresas han abocado su atención, su relación directa con el liderazgo y la diferenciación la han convertido en pieza fundamental para forjar la sostenibilidad y la rentabilidad para quienes participan en la carrera de la competitividad. Ello involucra una serie de factores que deben ser analizados a profundidad, para establecer estrategias de mercadeo estrechamente ligadas con la satisfacción del cliente, lo cual será el punto focal de las acciones a tomar que garanticen el regreso del cliente.

Esta forma de satisfacer al cliente se ha convertido en un fenómeno cada vez de mayor impacto, el camino hacia la excelencia del servicio no es un camino sencillo, requiere fortaleza y constancia para instalar en la empresa una cultura orientada a la satisfacción del cliente, ello implica mucho más que un departamento de atención al cliente, requiere una actitud crítica de toda la organización de la prestación del servicio, se trata de entender y respetar el punto de vista del cliente.

En este marco referencial, las organizaciones petroleras deben ser competitivas y ofrecer una entrega oportuna de productos o servicios de calidad, este nuevo entorno de exigencias ha comprometido a sus gerentes a optimizar todos sus procesos. Bajo tales consideraciones, la calidad del servicio representa una función clave para encauzar las operaciones y contribuir al logro de los objetivos de la organización, por ello, en ellas ha de concentrarse esfuerzos en el aumento de sus 
capacidades, evolucionando hacia la mejora de su eficiencia, que lleva a las mismas a la obtención de sus metas.

En este punto, la industria petrolera en Venezuela no debería quedar relegada del resto del mundo. Petróleos de Venezuela Sociedad Anónima (en adelante PDVSA), es la primera industria venezolana en el sector de hidrocarburos, que tiene como misión diseñar estrategias que permitan la recuperación eficiente y rentable para la reserva del mismo. Mediante la elaboración de planes y explotación, promoviendo el mejoramiento continuo para los procesos asociados, garantizando el manejo para la gestión con sentido del negocio, basados en el desarrollo del personal y tecnología, con el mayor grado de seguridad, protección ambiental, calidad, flexibilidad, dirigidos hacia la máxima satisfacción de sus clientes, la búsqueda permanente del éxito, es decir, satisfacer las necesidades energética de la sociedad.

En este marco de referencia, PDVSA es una corporación mundial de energía, propiedad del estado venezolano, encargada del desarrollo de la industria petrolera, petroquímica y carbonífera. Tiene la función de planificar, coordinar, supervisar, controlar las actividades de sus empresas operadoras más las filiales, tanto en Venezuela como en el exterior. Cabe resaltar que dentro de su estructura organizacional se ubican las empresas mixtas, que tienen como objetivo social el desarrollo de actividades primarias de exploración en busca de yacimientos de hidrocarburos, su extracción en estado natural, recolección, transporte y almacenamiento inicial, además de prestar servicio de ingeniería, construcción, reconstrucción y reparación para el desarrollo de proyectos vinculados con Costa Afuera.

Actualmente, en estas empresas se ha venido observando un mayor reconocimiento por parte de la gerencia, de la importancia que tiene la calidad de servicio para alcanzar un control eficiente del proceso operacional. Así que, de acuerdo a la información suministrada por el personal, existen fallas directas en cuanto a la manera como se ejecutan los procesos, dado que no se les brinda continuamente al personal adiestramiento para actualizar o mantener sus conocimientos en la manera de realizar un proceso de calidad, ajustado a las necesidades del cliente y a los requerimientos del entorno en el cual se desenvuelven, así como se refleja poco seguimiento a las actividades ejecutadas, lo cual afecta el logro de los objetivos.

Bajo esta óptica, vale resaltar la conveniencia de realizar un análisis de la calidad de servicio en las empresas mixtas de la industria petrolera del municipio Maracaibo. Para ello, la autora consideró importante abordar los elementos de la calidad del servicio, a fin de conocer las áreas débiles que requieran de mejora.

\section{Elementos de la calidad del servicio}

La evaluación de la calidad comprende cuatro elementos, según Gryna y otros (2007): el costo de mala calidad, posición en el mercado, cultura de la calidad en la organización, y operación del sistema de la calidad empresarial, los cuales, constituyen un análisis del estatus de la calidad de 
una empresa en términos de fortalezas, debilidades, oportunidades y amenazas (SWOT, por sus siglas en ingles) y llegan a ser una parte formal del análisis SWOT para las operaciones generales de una organización.

En este orden, González (2003), lo define como aquellas acciones que hacen que un producto o servicio cumpla con unos determinados requisitos de calidad, es decir, es el esfuerzo total para plantear, organizar, dirigir y controlar la calidad en un sistema de producción con el objetivo de dar al cliente productos con la calidad adecuada. Si estos requisitos de calidad reflejan completamente las necesidades de los clientes, se podrá decir que se cumple el aseguramiento de la calidad.

Ahora bien, para Evans y Lindsay (2008), el tener un sistema de evaluación de la calidad ayuda a mantener mejoras cotidianamente. Las empresas deben tener un muy buen sistema de evaluación de la calidad, que se enfoque en lograr productos y servicios que no tengan defectos, y que cumplan con los requerimientos o especificaciones. La evaluación de la calidad, según la norma ISO 9001: 2008, es un conjunto de acciones planificadas y sistemáticas, implementadas en el sistema de calidad, que son necesarias para proporcionar la confianza adecuada de que un producto satisfará los requisitos dados sobre la calidad.

A efectos de analizar la calidad del servicio, se asumen los elementos planteados por los autores citados, en este sentido, se visualiza que a través de evaluar los costos incurridos por la mala calidad del servicio prestado, la posición que ocupa la organización dentro de su mercado, la cultura de calidad manejada por los integrantes de la organización y la forma como opera el sistema de calidad interno a la organización, se pueden determinar los elementos para una evaluación precisa de la calidad de servicio en los procesos de evaluaciones económicas de contratos, en las empresas mixtas bajo estudio. De forma tal, los mismos se constituyen en los indicadores que conformarán esta dimensión de la variable, y conformarán parte del análisis de la calidad del servicio a través de la aplicación del instrumento de medición.

\section{Costo de la mala calidad}

Para Gryna y otros (2007), los costos de la mala calidad son aquellos costos en los que se incurren para determinar si la producción es aceptable, es decir la inversión que se hace para verificar el nivel de calidad del producto más la realizada para prevenir o corregir la ocurrencia de la no calidad, pero a estos se les suma cualquier otro costo en que incurre la empresa conjuntamente con el cliente porque la producción no cumplió las especificaciones. Cuatrecasas (2010) los define como aquellos que se derivan de la ausencia de calidad, se producen como resultado de los fallos o errores en el diseño, desarrollo, producción, y que puedan trascender o no hasta el cliente o consumidor.

También se incluyen los costos por falta de un adecuado servicio al cliente: posventa o reparaciones entre otros; que provocan una insatisfacción en las expectativas y necesidades que tiene el cliente. Como consecuencia de no alcanzar el nivel de calidad deseado, el cliente puede 
optar por productos o servicios de la competencia, arrastrando consigo a otros clientes potenciales.

En esta perspectiva, a juicio de Tamimi (2008), el costo de la mala calidad es la pérdida anual monetaria de los productos y procesos producentes de resultados contrarios a los objetivos de calidad. Asegura el autor citado que el costo de la mala calidad (COPQ, por sus siglas en inglés) recibe, apropiadamente, el nuevo nombre de costo por los procesos de mal desempeño. Esto es para enfatizar el hecho de que el costo de la mala calidad no se limita solo a esta, sino que es esencialmente el costo del cumplimiento aunado al inconveniente de los procesos.

Ahora bien, cuando se habla del costo de la mala calidad Howell (2006), afirma que son gastos extras causados por la entrega de bienes o servicios en mala calidad a los clientes. Para este autor estos gastos tienen dos fuentes: el fracaso de costos internos (por defectos de antes de que el cliente recibiera el producto) y el fracaso de costos externos (costos de después de que el cliente reciba el producto o servicio en malas condiciones). Realizar las reparaciones o la pérdida del negocio son ejemplos de los costos asociados con la mala calidad.

A efectos para el estudio, el costo de la mala calidad debe ser un factor de preocupación para cualquier empresa, en el sentido de que trae como consecuencia una mala imagen de la organización entre la cartera de clientes, adicional a que podrá afectar de manera directa su posicionamiento en relación con el resto de las empresas ofertantes de su mismo servicio y, como resultado, una disminución considerable de su rentabilidad. Se infiere entonces que altos costos asociados a mala calidad generan disminución de personal y acarrea un trabajo fuerte de reingeniería empresarial para determinar cuáles son las áreas de atención para reducir dichos costos, representando pérdidas directas sobre el capital financiero de la organización.

En el caso de los servicios, el costo de la mala calidad incluye los costos por falta de un adecuado servicio al cliente que provoca una insatisfacción en las expectativas y necesidades que tiene el cliente. Como consecuencia de no alcanzar el nivel de calidad deseado, el cliente puede optar por servicios de la competencia, arrastrando consigo a otros clientes potenciales.

\section{Posición del mercado}

Uno de los factores fundamentales en el éxito de los productos se relaciona a lograr un adecuado posicionamiento en mercados competitivos. En cierta forma, Miranda y otros (2007), hablan del posicionamiento como la manera como se dará a conocer el producto o servicio ofrecido y se pretende que sea percibido por el mercado meta. En este orden, para James (2006), estimar el costo de la mala calidad es una parte esencial de la evaluación, pero no es suficiente, también se necesita conocer donde se encuentra la empresa en cuestión de calidad en el mercado, con relación a la competencia. Este elemento de la evaluación de la calidad probará ser importante cuando se aumenten los ingresos por ventas. 
Ahora bien, a criterio de Riveros (2007), un mercado se compone de personas y organizaciones con necesidades, dinero que gastar y con el deseo de gastarlo. Sin embargo, dentro de la mayor parte de los mercados las necesidades de los compradores no son las mismas. Es por ello, que una empresa debe profundizar en el conocimiento de su mercado con el objeto de adaptar su oferta además de su estrategia de marketing a los requerimientos de éste. Por ende, según Miranda y otros (2007), la identificación y elección de los segmentos de mercado, plantea el problema de decidir la posición que desea la empresa ocupar en dichos mercados, es decir, elegir un posicionamiento para sus productos.

Por lo tanto, parecido al costo de la mala calidad como elemento para evaluar la calidad de servicio, el estudio de mercado da una instantánea noción de la posición de la empresa en relación con la competencia e identifica oportunidades y amenazas. En relación con este punto, el posicionamiento en el mercado de una empresa es un indicador directo de la calidad del servicio ofrecido al mercado donde se desenvuelve, siendo directamente proporcional lo uno con respecto a lo otro; es decir, mientras mayor es la calidad, más alto será el posicionamiento de la organización.

\section{Cultura de la calidad}

Para Cantú (2011) la cultura de la calidad en la organización es:

El conjunto de valores y hábitos que posee una persona, que complementados con el uso de prácticas o herramientas de calidad en el actuar diario, le permiten colaborar con su organización para afrontar los retos que se le presenten, en el cumplimiento de la misión de la organización. Valores y hábitos son aquellas impresiones profundas que se tiene sobre la forma en que se vive, sobre lo que se considera correcto o incorrecto (p. 85).

Al respecto, Ortega y Suarez (2009), plantean que los empleados de una organización tienen opiniones, creencias, tradiciones y prácticas que tienen que ver con la calidad, a este conjunto de características se le llama cultura de calidad de la empresa. Lograr un entendimiento de esta cultura debería ser parte de una evaluación de la calidad por dos razones: la cultura claramente tiene un impacto principal sobre los resultados de la calidad y conocer la cultura presente puede identificar barreras para desarrollar una estrategia e implementar un plan de acción basado en la evaluación de la calidad.

De acuerdo con Cantú (2011), para que el cambio cultural de una empresa tenga éxito, se debe de involucrar al mayor número de personas que tengan el perfil cultural deseado. Con esta afirmación, Ortega y Suarez (2009), consideran que se debe buscar, a través de exámenes psicométricos, individuos con ciertas similitudes en los procesos de pensamiento y de actuación siendo estos los que en un momento posterior, expandan el cambio en la organización, es decir, se 
deben de buscar los perfiles adecuados para que sean esas personas los iniciadores del cambio en la organización. Adicional a esto, afirman los autores citados, al momento de planear el cambio dentro de la organización es imprescindible que se cambien los paradigmas que todavía se encuentran presentes en las organizaciones.

En esta perspectiva, para Guajardo (2008), es importante reconocer que en una empresa las opiniones y prácticas diversas acerca a la calidad que poseen los empleados forman la cultura de la calidad, donde su entendimiento debe formar parte de la evaluación de la calidad. En esta perspectiva, a juicio de Gryna y col. (2007), las opiniones, creencias, tradiciones, valores y conductas de los empleados de una organización conforman lo que en gestión de la calidad se ha llamado la cultura de calidad de la empresa.

De esta forma, la puesta en práctica de un plan de cultura de calidad en la empresa no es fácil; se requiere personal altamente comprometido con la empresa con los valores a implantar $y$, altamente capacitados en el trato de personal. Además es muy costosa, requiere de la participación activa por parte de la administración de todos los niveles; además, esta tiende a disminuir con el paso del tiempo por falta de disciplina de los empleados.

Sin embargo, a pesar de las dificultades, es necesario hacer un esfuerzo para implantarla, ya que la exigencia de los clientes crece continuamente obligando a que los empleados, además de hacer bien el trabajo, tengan ciertas características orientadas a la calidad de trabajo y de vida, lo cual es buscado por las empresas que buscan la mejora continua en la calidad.

\section{Operación del sistema}

A juicio de Cantú (2011), se ha considerado como el cuarto elemento de evaluación de la calidad a la valoración de las operaciones del sistema o actividades actuales relacionadas con la calidad dentro de la organización. Dicha evaluación, podría cubrir un amplio rango de ámbitos, podría ser global para unas cuantas actividades o exámenes desde superficiales a detallados.

En este orden de ideas, para Ortega y Suarez (2009), la evaluación de las operaciones del sistema de calidad puede tomar dos formas:

- Evaluaciones que se enfocan en los resultados de la satisfacción del cliente, pero incluyen evaluación del sistema actual de actividades relacionadas con la calidad.

- Evaluaciones que se enfocan en la valoración del actual sistema de calidad, con poco énfasis en los resultados de la satisfacción del cliente.

En cualquiera de los dos casos, la evaluación puede ser conducida por la misma organización (autoevaluación) o por una organización externa. También puede ser llevada a cabo con o sin criterios definidos explícitamente. Ambos tipos de evaluación pueden identificar oportunidades de 
mejora al comparar las actividades actuales de la calidad con un modelo de sistemas aceptado, tal como el ISO 9000.

En este sentido, a fines de la investigación, las evaluaciones de las operaciones del sistema, como elemento de evaluación de la calidad de servicio, deben ser prioridad de las empresas mixtas, en el sentido de que sus procesos de evaluaciones económicas de contratos deben ser vistos desde la óptica de la calidad de servicio, por ello estas evaluaciones a sus procesos les permitirán a los gerentes tomar las acciones preventivas o correctivas que se ameriten para mejorar la calidad del servicio prestado.

Por lo tanto, los elementos para la valoración de la calidad del servicio permiten a las unidades de producción modificar sus planes estratégicos teniendo en cuenta el costo de la mala calidad, es decir, el hacer las cosas mal, repetirlas varias veces al no cumplir con los requisitos del cliente, como por ejemplo, ciñéndose al caso en estudio, realizar mal algún documento vital para las evaluaciones de contratos, los cuales se realizan varias veces, debido a diferencia de criterios, combinado al no cumplimiento de las expectativas de los clientes. En tal sentido, se debe propiciar en todo el personal de las organizaciones una cultura de calidad claramente definida en función del cumplimiento de los objetivos, evaluando las oportunidades y amenazas para alcanzar un posicionamiento positivo en el mercado.

\section{MÉTODO}

$\mathrm{L}$ a metodología utilizada en la investigación fue de tipo descriptiva, con diseño no experimental, transeccional y de campo. La población quedó conformada por las gerencias de contratación de las tres empresas mixtas de la industria petrolera del municipio Maracaibo, siendo ellas: Petroboscan, Petroindependente y Petrowayu; cuyos sujetos informantes fueron los gerentes, líderes y supervisores, tal como se describe en el cuadro 1.

Cuadro 1. Distribución de la población

\begin{tabular}{|c|c|c|c|c|}
\hline Empresa & Gerentes & Lideres & Supervisores & Total \\
\hline Petroboscan & 1 & 2 & 4 & 7 \\
\hline Petroindependente & 1 & 1 & 4 & 6 \\
\hline Petrowayu & 1 & 2 & 4 & 7 \\
\hline Total & 3 & 5 & 12 & 20 \\
\hline
\end{tabular}

Para la recolección de datos se empleó la encuesta mediante la aplicación de un cuestionario, el mismo quedó conformado por doce ítems con escala tipo Likert. La validez se realizó a través del juicio de expertos, y para calcular su confiabilidad se empleó el método del Coeficiente Alfa de Cronbach, cuyo resultado fue 0,91 indicando muy alta confiabilidad. 
El análisis de los datos se realizó mediante la estadística descriptiva, con base en la media aritmética, apoyada en las frecuencias relativas. Para tal efecto, se diseñó un (1) baremo para el análisis de la media aritmética, el mismo se visualiza en el cuadro 2.

Cuadro 2. Baremo para la interpretación de la media aritmética

\begin{tabular}{lcc}
\hline \multicolumn{1}{c}{ Alternativa } & Intervalo & Categoría \\
\hline Totalmente de acuerdo & $4.21-5.00$ & Muy alta presencia \\
De acuerdo & $3.43-4.20$ & Alta presencia \\
Indiferente & $2.62-3.42$ & Moderada presencia \\
Desacuerdo & $1.81-2.61$ & Baja presencia \\
Totalmente desacuerdo & $1.00-1.80$ & Muy baja presencia \\
\hline
\end{tabular}

Para el análisis de las frecuencias se consideró conveniente fijar un baremo de interpretación, el cual se recoge en el cuadro 3.

Cuadro 3. Tendencia de análisis para la interpretación del porcentaje

\section{TENDENCIA}

Tendencia Positiva cuando Ta $+\mathrm{Da} \geq 75 \%$

Tendencia Neutral cuando $45 \% \leq \mathrm{Ta}+\mathrm{Da}<75 \%$

Tendencia Negativa cuando Ta $+\mathrm{Da}<45 \%$

\section{RESULTADOS}

$\mathrm{S}$ e presentó el análisis para los elementos de la calidad de servicio partiendo de los cuatro indicadores: costo de la mala calidad, posición del mercado, cultura de la calidad y operación del sistema. Comenzando con el indicador costo de la mala calidad, se tiene en la tabla 1 los resultados obtenidos, reflejándose, con una tendencia positiva de las respuestas (75\%), que los encuestados están totalmente de acuerdo y de acuerdo en otorgarle alta presencia $(4,15)$ al costo de la mala calidad como elemento para evaluar la calidad de servicio prestado, implicando una fortaleza en la calidad de servicio de estas empresas.

Al observar al detalle los resultados, se puede apreciar que un $75 \%$ de los encuestados, concentraron sus respuestas en las opciones totalmente de acuerdo y de acuerdo marcando una tendencia positiva para todos los ítems involucrados a este indicador, al mismo tiempo que categorizaron con alta presencia cada uno de ellos. Por lo cual, se pudo evidenciar que en las empresas mixtas estudiadas se realizó con alta presencia el que: cuantifiquen la envergadura de la 
calidad en términos monetarios $(4,1)$, identifiquen con regularidad las oportunidades principales para la reducción de costos $(4,15)$, e identifiquen las oportunidades para reducir el descontento de los clientes $(4,2)$.

Los resultados alcanzan alta congruencia con lo manifestado por Gryna y otros (2007), Tamimi (2008) y Howel (2006) cuando coinciden en que las empresas estiman el costo de la mala calidad, entre otras razones, para: cuantificar la envergadura del problema de la calidad en lenguaje monetario, identificar las oportunidades principales para la reducción de costos, identificar las oportunidades para reducir el descontento de los clientes y las amenazas asociadas a la posibilidad de venta del producto.

Tabla 1. Indicador: Costos de la mala calidad

\begin{tabular}{|c|c|c|c|c|c|c|}
\hline \multicolumn{7}{|c|}{ Ítems } \\
\hline \multirow{3}{*}{\multicolumn{7}{|c|}{$\begin{array}{l}\text { 1. Cuantifican la envergadura de la calidad de servicio en lenguaje monetario. } \\
\text { 2. Las oportunidades principales para la reducción de costos son identificadas con regularidad. } \\
\text { 3. Identifican las oportunidades para reducir el descontento de los clientes. }\end{array}$}} \\
\hline & & & & & & \\
\hline & & & & & & \\
\hline \multirow{3}{*}{ Categoría } & \multicolumn{6}{|c|}{ Ítems/Frecuencias } \\
\hline & \multicolumn{2}{|c|}{1} & \multicolumn{2}{|c|}{2} & \multicolumn{2}{|c|}{3} \\
\hline & FA & FR & FA & FR & FA & $\mathbf{F R}$ \\
\hline Totalmente de cuerdo & 11 & $55 \%$ & 12 & $60 \%$ & 11 & $55 \%$ \\
\hline De acuerdo & 4 & $20 \%$ & 3 & $15 \%$ & 4 & $20 \%$ \\
\hline Indiferente & 2 & $10 \%$ & 2 & $10 \%$ & 3 & $15 \%$ \\
\hline Desacuerdo & 2 & $10 \%$ & 2 & $10 \%$ & 2 & $10 \%$ \\
\hline \multicolumn{7}{|l|}{ Totalmente } \\
\hline desacuerdo & 1 & $5 \%$ & 1 & $5 \%$ & 0 & $0 \%$ \\
\hline Total & 20 & $100 \%$ & 20 & $100 \%$ & 20 & $100 \%$ \\
\hline $\mathrm{Ta}+\mathrm{Da}$ & \multicolumn{2}{|c|}{$75 \%$} & \multicolumn{2}{|c|}{$75 \%$} & \multicolumn{2}{|c|}{$75 \%$} \\
\hline Tendencia Ítems & \multicolumn{2}{|c|}{ Positiva } & \multicolumn{2}{|c|}{ Positiva } & \multicolumn{2}{|c|}{ Positiva } \\
\hline Alternativa Indicador & \multicolumn{6}{|c|}{$75 \%$} \\
\hline Tendencia Indicador & \multicolumn{6}{|c|}{ Positiva } \\
\hline
\end{tabular}

\section{Promedio de las respuestas codificadas}

Categoría

Promedio Indicador

Categoría

\section{Ítems}

\section{1}

2

3

4,10

4,15

4,20

Alta presencia

Alta presencia

Alta presencia

4,15

Alta presencia 
Se evidencia de estos hallazgos que, para las empresas mixtas bajo estudio el costo de la mala calidad ocupa alta presencia como elemento para evaluar la calidad de servicio, implicando una fortaleza para estas empresas. Se deduce entonces, que estas empresas se han concientizado en cuanto a la importancia de este costo dentro de la evaluación de la calidad de servicio prestado, atribuyéndole a estos costos la falta de un adecuado servicio al cliente, lo cual puede provocar una insatisfacción en las expectativas y necesidades que tiene el mismo.

El siguiente indicador que se analizó, en esta dimensión, es el denominado: posición del mercado. Los resultados relativos a él, se resumen en la tabla 2, observándose que, en promedio, el $70 \%$ de los encuestados se posicionó en las opciones de respuesta totalmente de acuerdo y de acuerdo, demarcando una tendencia neutral en dichas respuestas; otorgándole, además, alta presencia $(4,08)$ como elemento para evaluar la calidad de servicio, implicando una fortaleza para las empresas estudiadas.

Tabla 2. Indicador: Posición del mercado

\section{Ítems}

4. Conocen donde se posicionan en cuestión de calidad de servicio en el mercado.

5. Se determinan las oportunidades de mercado.

6. Poseen conocimiento de su mercado con el objeto de adaptar su servicio además de su estrategia.

\section{Categoría}

Totalmente de cuerdo

De acuerdo

Indiferente

Desacuerdo

Totalmente desacuerdo

Total

$\mathrm{Ta}+\mathrm{Da}$

Tendencia Ítems

Alternativa Indicador

Tendencia Indicador

Promedio de las

respuestas codificadas

Categoría

Promedio Indicador

Categoría
4

$$
\text { FA }
$$

12

2

4

4

2

0

20

$70 \%$

Neutral

\section{Ítems/Frecuencias}

5

$\begin{array}{ccc}\text { FA } & \text { FR } \\ 9 & & 45 \% \\ 5 & & 25 \% \\ 3 & & 15 \% \\ 3 & & 15 \% \\ 0 & & 0 \% \\ 20 & & 100 \% \\ & \mathbf{7 0 \%} & \end{array}$

Neutral

$70 \%$
6

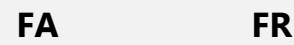

10

$50 \%$

$20 \%$

$15 \%$

$15 \%$

$0 \%$

$100 \%$

Neutral 
Adicionalmente, la tabla muestra como el $70 \%$ de las respuestas emitidas en las opciones positivas indicaron una tendencia neutral para todos los reactivos medidos. Aunado a esto, todos los ítems se posicionaron en la categoría de alta presencia $(4,20 ; 4,00 ; 4,05$; respectivamente) afirmando que los siguientes aspectos presentan alta presencia: conocen donde se posicionan en cuestión de calidad de servicio en el mercado, determinan las oportunidades de mercado, y poseen conocimiento de su mercado con el objeto de adaptar su servicio además de su estrategia.

Los valores alcanzados para el indicador validan lo expuesto por James (2006), para quien estimar el costo de la mala calidad es una parte esencial de la evaluación, pero no es suficiente, también se necesita conocer donde se encuentra la empresa en cuestión de calidad en el mercado, con relación a la competencia. Asimismo, se tiene coincidencia con Riveros (2007), cuando afirma que la posición que alcance una organización de servicios dentro de su mercado operativo, dependerá en gran medida de la calidad de su servicio.

Lo expuesto, permite deducir que en las empresas mixtas, objeto de estudio, la posición del mercado es tomada con alta presencia para evaluar la calidad de servicio prestada.

Ahora bien, en esta exposición de resultados, le corresponde el turno al indicador cultura de la calidad, como elemento para evaluar la calidad de servicio en las empresas objeto de estudio. Se aprecia en la tabla 3, que este indicador alcanzó una tendencia neutral en las respuestas dadas por los encuestados al obtener un promedio de $66,67 \%$, aun cuando muestra alta presencia $(3,92)$.

Al detallar los resultados por ítems, se evidenció que las actividades referidas en todos ellos se categorizaron con alta presencia, referidas a si aplican prácticas relacionadas con la calidad de servicio; identifican barreras para desarrollar una estrategia implementada en un plan de acción basado en la evaluación de la calidad de servicio; y evalúan la cultura de la calidad de servicio mediante el uso de cuestionarios para encuestar a los empleados.

Los resultados alcanzados validan lo planteado por Guajardo (2008), para quien es importante reconocer que en una empresa las opiniones y prácticas diversas acerca a la calidad que poseen los empleados forman la cultura de la calidad, donde su entendimiento debe formar parte de la evaluación de la calidad. También validan a Gryna y otros (2007), cuando afirman que las opiniones, creencias, tradiciones, valores y conductas de los empleados de una organización conforman lo que en gestión de la calidad se ha llamado la cultura de calidad de la empresa.

Tabla 3. Indicador: Cultura de la calidad

\section{Ítems}

7. Aplican prácticas relacionadas con la calidad de servicio.

8. Identifican barreras para desarrollar una estrategia, implementada en un plan de acción basado en la evaluación de la calidad del servicio.

9. La cultura de la calidad de servicio se evalúa mediante el uso de cuestionarios para encuestar a los empleados. 


\begin{tabular}{|c|c|c|c|c|c|c|}
\hline \multirow{3}{*}{ Categoría } & \multicolumn{6}{|c|}{ Ítems/Frecuencias } \\
\hline & \multicolumn{2}{|c|}{7} & \multicolumn{2}{|c|}{8} & \multicolumn{2}{|c|}{9} \\
\hline & FA & FR & FA & $\mathbf{F R}$ & FA & FR \\
\hline Totalmente de cuerdo & 10 & $50 \%$ & 11 & $55 \%$ & 8 & $40 \%$ \\
\hline De acuerdo & 4 & $20 \%$ & 3 & $15 \%$ & 4 & $20 \%$ \\
\hline Indiferente & 4 & $20 \%$ & 4 & $20 \%$ & 1 & $5 \%$ \\
\hline Desacuerdo & 2 & $10 \%$ & 2 & $10 \%$ & 4 & $20 \%$ \\
\hline Totalmente desacuerdo & 0 & $0 \%$ & 0 & $0 \%$ & 3 & $15 \%$ \\
\hline Total & 20 & $100 \%$ & 20 & 100 & 20 & $100 \%$ \\
\hline $\mathrm{Ta}+\mathrm{Da}$ & \multicolumn{2}{|c|}{$70 \%$} & \multicolumn{2}{|c|}{$70 \%$} & \multicolumn{2}{|c|}{$60 \%$} \\
\hline Tendencia Ítems & \multicolumn{2}{|c|}{ Neutral } & \multicolumn{2}{|c|}{ Neutral } & \multicolumn{2}{|c|}{ Neutral } \\
\hline Alternativa Indicador & \multicolumn{6}{|c|}{$66,67 \%$} \\
\hline Tendencia Indicador & \multicolumn{6}{|c|}{ Neutral } \\
\hline
\end{tabular}

\begin{tabular}{lccc} 
& \multicolumn{3}{c}{ Ítems } \\
Promedio de las & $\mathbf{7}$ & $\mathbf{8}$ & $\mathbf{9}$ \\
\cline { 2 - 4 } respuestas codificadas & 4,10 & 4,15 & 3,50 \\
& Alta presencia & Alta presencia & Alta presencia \\
Categoría & & $\mathbf{3 , 9 2}$ & \\
Promedio Indicador & & Alta presencia & \\
Categoría & &
\end{tabular}

En el caso de las empresas mixtas bajo estudio, se mostró la cultura de calidad con alta presencia como elemento para evaluar la calidad, De esta forma, la puesta en práctica de un plan de cultura de calidad en la empresa no es fácil; dado a que, se requiere personal altamente comprometido con la empresa con los valores a implantar $y$, altamente capacitados en el trato de personal.

Sin embargo, a pesar de las dificultades, es necesario hacer un esfuerzo para implantarla, ya que la exigencia de los clientes crece continuamente obligando a que los empleados, además de hacer bien el trabajo, tengan ciertas características orientadas a la calidad de trabajo y de vida, lo cual es buscado por las empresas en la mejora continua en la calidad.

Para finalizar, el análisis de los indicadores utilizados en la dimensión elementos para la evaluación de la calidad, se tiene lo referente al indicador operación del sistema. Para ello se mostró la tabla 4, contentiva de los hallazgos resultantes de la aplicación del instrumento de recolección de datos para el indicador en cuestión. Se apreció entonces, que el indicador alcanza un promedio de respuestas en las opciones totalmente de acuerdo y de acuerdo de $76,67 \%$, indicando tendencia positiva de las respuestas dadas, acompañado de alta presencia de este indicador $(4,18)$.

Los ítems utilizados para medir el indicador mostraron el siguiente comportamiento: para el ítem 10, el 70\% de los encuestados se muestran totalmente de acuerdo y de acuerdo, otorgándole 
tendencia neutral a las respuestas dadas, de catalogar con alta presencia $(3,95)$ al que las evaluaciones de las actividades actuales de calidad de servicio se enfocan en los resultados de la satisfacción del cliente.

Mientras que los ítems 11 y 12 ostentan muy alta presencia (4,35 y 4,25); indicando que los encuestados consideran con muy alta presencia el que las oportunidades de mejora se establecen al comparar las actividades actuales de la calidad de servicio con un modelo de sistema aceptado como el ISO 9000, y las evaluaciones de las actividades actuales de calidad de servicio se enfocan en los resultados de la valoración del actual sistema. En ambos casos se muestra tendencia positiva en las respuestas emitidas.

Estos resultados validan lo expresado por Ortega y Suarez (2009), para quienes la evaluación de las operaciones del sistema de calidad puede tomar dos formas: evaluaciones que se enfocan en los resultados de la satisfacción del cliente, pero incluyen una evaluación del sistema actual de las actividades relacionadas con la calidad, y evaluaciones que se enfocan en la valoración del actual sistema de calidad, con poco énfasis en los resultados de la satisfacción del cliente. Ambos tipos de evaluación pueden identificar oportunidades de mejora, al comparar las actividades actuales de calidad con un modelo de sistema aceptado, tal como el ISO 9000.

Tabla 4. Indicador: Operación del sistema

\section{Ítems}

10. Las evaluaciones de las actividades actuales de la calidad del servicio se enfocan en los resultados de la satisfacción del cliente.

11. Las oportunidades de mejora se establecen al comparar las actividades actuales de la calidad de servicio con un modelo de sistema aceptado, tal como el ISO 9000.

12. Las evaluaciones de las actividades actuales de calidad de servicio se enfocan en los resultados de la valoración del actual sistema.

\begin{tabular}{|c|c|c|c|c|c|c|}
\hline \multirow{3}{*}{ Categoría } & \multicolumn{6}{|c|}{ Ítems/Frecuencias } \\
\hline & \multicolumn{2}{|c|}{10} & \multicolumn{2}{|c|}{11} & \multicolumn{2}{|c|}{12} \\
\hline & FA & FR & FA & FR & FA & FR \\
\hline Totalmente de cuerdo & 8 & $40 \%$ & 11 & $55 \%$ & 11 & $55 \%$ \\
\hline De acuerdo & 6 & $30 \%$ & 6 & $30 \%$ & 4 & $20 \%$ \\
\hline Indiferente & 3 & $15 \%$ & 2 & $10 \%$ & 4 & $20 \%$ \\
\hline Desacuerdo & 3 & $15 \%$ & 1 & $5 \%$ & 1 & $5 \%$ \\
\hline Totalmente desacuerdo & 0 & $0 \%$ & 0 & $0 \%$ & 0 & $0 \%$ \\
\hline Total & 20 & $100 \%$ & 20 & $100 \%$ & 20 & $100 \%$ \\
\hline $\mathrm{Ta}+\mathrm{Da}$ & \multicolumn{2}{|c|}{$70 \%$} & \multicolumn{2}{|c|}{$85 \%$} & \multicolumn{2}{|c|}{$75 \%$} \\
\hline Tendencia Ítems & \multicolumn{2}{|c|}{ Neutral } & \multicolumn{2}{|c|}{ Positiva } & \multicolumn{2}{|c|}{ Positiva } \\
\hline Alternativa Indicador & \multicolumn{6}{|c|}{$76,67 \%$} \\
\hline Tendencia Indicador & \multicolumn{6}{|c|}{ Positiva } \\
\hline
\end{tabular}




\begin{tabular}{lccc}
\hline Promedio de las respuestas & $\mathbf{1 0}$ & Ítems & $\mathbf{1 2}$ \\
codificadas & 3,95 & $\mathbf{1 1}$ & 4,25 \\
& Alta presencia & Muy alta presencia & Muy alta presencia \\
Categoría & & $\mathbf{4 , 1 8}$ & \\
Promedio Indicador & & Alta presencia & \\
Categoría & & \\
\hline
\end{tabular}

Las empresas mixtas analizadas muestran alta presencia de la operación del sistema como elemento de evaluación de la calidad de servicio. De esta forma, deben ser prioridad de las empresas mixtas, en el sentido de que sus procesos de evaluaciones económicas de contratos deben ser vistos desde la óptica de la calidad de servicio, por ello estas evaluaciones a sus procesos les permitirán a los gerentes tomar las acciones preventivas o correctivas que se ameriten para mejorar la calidad del servicio prestado.

Ya analizados cada uno de los indicadores considerados en la dimensión denominada elementos para la evaluación de la calidad, se pasa a verificar los resultados arrojados para esta dimensión. En la tabla 5 se observa un promedio de respuestas en la categoría totalmente de acuerdo y de acuerdo del $72,09 \%$, indicando tendencia neutral en las respuestas obtenidas, con una media de 4,08 ubicando a esta dimensión en la categoría de alta presencia en la calidad de servicio en las empresas bajo estudio. Se evidenció que todos los indicadores ostentaron la categoría de alta presencia, con tendencias positivas para el costo de la mala calidad y operación del sistema; mientras que la posición del mercado y cultura de la calidad se sitúan con tendencias neutrales.

Tabla 5. Dimensión: Elementos de la calidad de servicio

\begin{tabular}{lcccc}
\hline \multicolumn{1}{c}{ Indicador } & Ta + Da & Categoría & Media & Categoría \\
\hline Costos de la mala calidad & $75 \%$ & Positiva & 4,15 & Alta presencia \\
Posición del mercado & $70 \%$ & Neutral & 4,08 & Alta presencia \\
Cultura de la calidad & $66,67 \%$ & Neutral & 3,92 & Alta presencia \\
Operación del sistema & $76,67 \%$ & Positiva & 4,18 & Alta presencia \\
\hline \multicolumn{1}{c}{ Promedio } & $\mathbf{7 2 , 0 9 \%}$ & Neutral & $\mathbf{4 , 0 8}$ & Alta presencia \\
\hline
\end{tabular}

Los resultados de alta presencia de la dimensión elementos de la calidad, validan lo expuesto por la norma ISO 9001: 2008, donde se establece que la evaluación de la calidad implica un conjunto de acciones planificadas y sistemáticas, implementadas en el sistema de calidad, necesarias para proporcionar la confianza adecuada de que un producto o servicio satisfará los requisitos dados sobre la calidad. 
También validan a González (2003), quien la define como aquellas acciones que hacen que un producto o servicio cumpla con unos determinados requisitos de calidad, es decir, es el esfuerzo total para plantear, organizar, dirigir y controlar la calidad en un sistema de producción con el objetivo de dar al cliente productos o servicios con la calidad adecuada. Si estos requisitos de calidad de servicio reflejan completamente las necesidades de los clientes, se cumple el aseguramiento de la calidad.

Con base a estas afirmaciones de los autores citados, se deduce que las empresas mixtas estudiadas, dado que se tiene alta presencia de los elementos de evaluación de la calidad, se posicionan con una fortaleza en el aseguramiento de la calidad del servicio prestado. Por ende, se afirma que a través de la evaluación de los costos incurridos por la mala calidad del servicio prestado, la posición que ocupa la organización dentro de su mercado, la cultura de calidad manejada por los integrantes de la organización y la forma como opera el sistema de calidad interno a la organización, se pudo determinar los elementos para una evaluación precisa de la calidad de servicio en los procesos de evaluaciones económicas de contratos, en las empresas mixtas bajo estudio.

\section{CONCLUSIÓN}

0 e determinaron los elementos de la calidad del servicio en las empresas mixtas venezolanas, siendo ellos: costo de la mala calidad, posición del mercado, cultura de la calidad y operación del sistema. Destacándose en los resultados, que esta dimensión arribo a la categoría de alta presencia, sin alcanzar la máxima categoría del baremo (muy alta presencia), dado que todos los indicadores clasificaron con la categoría de alta presencia.

Aun cuando las empresas analizadas ostentan alta presencia de los elementos considerados para la evaluación de la calidad, se tienen que revisar las acciones estratégicas que conllevan a la realización de las actividades medidas por los ítems utilizados. Así las cosas, es importante:

En cuanto al costo de la mala calidad:

- Velar porque se cuantifique en términos monetarios la envergadura de la calidad. Esto les permitirá medir el desempeño, indicando dónde se debe llevar a cabo una acción correctiva.

- Garantizar que sean identificadas con regularidad las oportunidades principales para la reducción de costos.

- Implementar la identificación de las oportunidades para reducir el descontento de los clientes, como medida de reducción de costos.

- Respecto a la posición del mercado, es importante: 
- Aplicar de forma periódica estudios de mercado para conocer donde se posicionan en cuestión de calidad de servicio en el mercado, dado que la posición en el mercado es uno de los indicadores que le permite evaluar la calidad del servicio.

- Garantizar que se determinen las oportunidades de mercado.

- Asegurar el conocimiento de su mercado a objeto de adaptar su servicio así como su estrategia.

- Implementar como política el que se utilice como indicador, para evaluar la calidad de servicio, la posición en el mercado. Esto permitirá reflexionar que la posición en el mercado es uno de los indicadores idóneos que permite a la empresa evaluar su calidad de servicio.

- Para la cultura de la calidad, se debe:

- Implementar como política el que se apliquen prácticas relacionadas con la calidad de servicio.

- Velar porque se identifiquen las barreras para desarrollar una estrategia, implementada en un plan de acción basado en la evaluación de la calidad del servicio.

- Garantizar que dentro de la evaluación de la cultura de la calidad de servicio se realicen encuestas a los empleados.

Finalmente, en relación a la operación del sistema, se requiere:

- Implementar como política que la evaluación de las actividades actuales de la calidad del servicio, se enfoquen en los resultados de la satisfacción del cliente. Se debe considerar que las organizaciones deben trabajar en pro de la satisfacción total de sus clientes, mediante un proceso de mejora continua aplicado a los sistemas de calidad, e implementar normas estandarizadas para lograr la calidad máxima de los productos o servicios que ofrecen.

\section{REFERENCIAS}

Cantú, J. (2011). Desarrollo de una cultura de calidad. Segunda edición. Editorial McGraw-Hill Latinoamericana. México

Cuatrecasas, L. (2010). Lean management, la gestión competitiva por excelencia: implantación Progresiva en 7 etapas Editorial Profit. Barcelona

Evans, J., y Lindsay, W. (2008). Administración y control de la calidad. Séptima edición. Editorial Cengage Learning Editores. México

González, F. (2003) ISO 9000:2000. Metodología avanzada para la implantación de sistemas de calidad. Un enfoque hacia los procesos del negocio. Segunda edición. Producciones KAROL, C.A. Mérida. Venezuela
Grande, I. (2005). Marketing de los servicios. Cuarta Edición. Editorial ESIC. España

Gryna, F.; Chua, R., y Defeo, J. (2007). Método Juran. Análisis y planeación de la calidad. Quinta Edición. McGraw-Hill. México

Guajardo, E. (2008). Administración de la calidad total. Conceptos y enseñanzas de los grandes maestros de la calidad. Tercera edición. Editorial PAX. México

Howell, M. (2006). Medición del desempeño accionable: una clave para el éxito. Milwaukee: ASQ Quality Press. New York

Ivancevich J.; Gibson, J., y Donnelly, J. (2005). Las organizaciones: comportamiento, estructura $y$ 
procesos. Octava Edición. Editorial Mc Graw Hill. Chile

James, P. (2006). Gestión de la calidad total. Un texto introductorio. Cuarta edición. Editorial Prentice Hall. España

Miranda, F.; Chamorro, A., y Rubio, S (2007) Introducción a la gestión de la calidad. Editorial Delta Publicaciones. Madrid, España

Ortega, M., y Suarez, M. (2009). Manual de evaluación de la calidad del servicio. Estrategias para su aplicación. 2da edición. Editorial Médica Panamericana. México

Riveros, P. (2007). Sistema de gestión de la calidad del servicio. Sea líder en mercados altamente competitivos. Tercera edición. Ediciones ECOE. Bogotá. Colombia

Tamimi, A. (2008). Cuantificación de los efectos de la calidad de inversión Disponible en: http://books.google.co.ve/books 